\title{
Risk of New Hospitalization Post-COVID-19 Infection for Non-COVID-19 Conditions
}

\author{
Arch G. Mainous III, PhD, Benjamin J. Rooks, MS, and Frank A. Orlando, MD
}

Introduction: Reports of post-acute sequelae of COVID-19 continue to emerge, but it remains unclear how the severity of a patient's COVID-19 infection affects risk for future hospitalizations for non-COVID-19 problems.

Methods: An analysis of electronic health records (EHR) was performed for a cohort of 10,646 patients who were followed for 6 months post-COVID-19 episode at 1 health system. COVID-19-positive patients were classified as severe if they were hospitalized within the first 30 days of their initial positive test. Assessment of hospitalizations overall and conditions that could be seen as complications of COVID-19 (cardiovascular, respiratory, and clotting diagnoses) was assessed. The 6-month risk of a new hospitalization was assessed in both unadjusted and adjusted Cox regressions.

Results: Of the 10,646 patients included in this cohort,114 had severe COVID-19, 211 had mild/ moderate COVID-19, and 10,321 were COVID-19 negative. After adjustment for potential confounding variables, there was no significantly increased risk in future hospitalization for any condition for patients who were COVID-19 positive versus those who were COVID-19 negative (HR, 1.31; 95\% CI, 0.98-1.74). In adjusted analyses, individuals with severe COVID-19 had an increased risk of hospitalization for potential complications compared with both mild/moderate COVID-19 (HR, 2.20; 95\% CI, 1.13-4.28) and COVID-19 negative patients (HR, 2.24; 95\% CI, 1.52-3.30).

Discussion: Patients with a severe COVID-19 episode were at greater risk for future hospitalizations. This study reinforces the importance of preventing infection in patients at higher risk for severe CoVID-19 cases. ( $\mathrm{J}$ Am Board Fam Med 2021;34:907-913.)

Keywords: Cohort Studies, COVID-19, Electronic Health Records, Hospitalization, Respiration Disorders

\section{Introduction}

By April 2021, Coronavirus disease 2019 (COVID-19) has resulted in over 133 million cases and 2.8 million deaths worldwide, with more than 30 million cases and 560,000 deaths in the United States alone. ${ }^{1}$ The clinical spectrum of COVID-19 infection ranges from asymptomatic infection to severe disease requiring admission to intensive care and even death. Examples of risk

This article was externally peer reviewed.

Submitted 19 April 2021; revised 13 May 2021; accepted 13 May 2021.

From the Department of Community Health and Family Medicine, University of Florida, Gainesville, FL (AGM, BJR, FAO); Department of Health Services Research Management, and Policy, University of Florida, Gainesville, FL (AGM).

Funding: We acknowledge the University of Florida Integrated Data Repository (IDR) and the UF Health Office of the Chief Data Officer for providing the analytic data set for this project. Additionally, the Research reported in this publication was supported by the National Center for Advancing Translational Sciences of the National Institutes of Health under University of Florida Clinical and Translational Science Awards UL1TR000064 and UL1TR001427. factors for severe COVID-19 include hypertension, diabetes, and obesity. ${ }^{2}$ Millions of people globally have "recovered." Recovery from COVID-19 is variable, and the post-acute complications can affect multiple organ systems and persist for many months. ${ }^{3}$ Many individuals experience persistent symptoms that impact their health-related quality of life, with some even reporting post-traumatic stress disorder. ${ }^{3-7}$

Severe complications like post-acute thrombosis, respiratory failure, and cardiac and vascular damage may increase the likelihood of future morbidity and mortality in recovered COVID-19 patients. ${ }^{8-10}$ The data from cohort studies investigating these long-term complications post COVID-19 infection is quite limited, and studies mainly focus on complications

Conflict of interest: The authors have no conflicts of interest. Corresponding author: Arch G. Mainous III, PhD, Department of Health Services Research, Management and Policy, University of Florida, Health Sciences Center, PO Box 100195, Gainesville, FL, Telephone: 352-273-6073 (Email: arch.mainous@ufl.edu). 
leading to readmission rather than post-acute complications. ${ }^{11-13}$ Therefore, it is unclear if the likelihood of future hospitalization for non-COVID-19 conditions is greater in post-COVID-19 patients than COVID-19-negative patients.

We investigated post-COVID-19 hospitalizations for specific non-COVID-19 conditions that also represent the common acute complications of severe COVID-19. We studied a longitudinal cohort of patients who tested either positive or negative for COVID-19 as determined by polymerase chain reaction (PCR) testing within a large health care system.

\section{Methods}

The data for this project comes from a deidentified research databank containing electronic health records (EHR) of patients tested for or diagnosed with COVID-19 in any setting in the University of Florida (UF) Health system. Usage of the databank for research is not considered human subjects research, and IRB review was not required to conduct this study.

\section{Definition of Cohort}

The cohort for this study consisted of all adult patients aged 18 years and older who were tested for COVID-19 between January 1, 2020, and July 5, 2020, within the UF Health system, in any encounter type (ambulatory, emergency department [ED], inpatient, etc). The databank contained EHR data for all patients in the current cohort through December 30, 2020. COVID-19 diagnosis was validated by PCR. Baseline dates for COVID-19-positive patients were established at the date of their earliest recorded PCRconfirmed positive COVID-19 test, and baseline for COVID-19-negative patients was assessed at the earliest recorded negative COVID-19 test. Each patient was only included once in the analysis. For patients with multiple COVID-19 tests, if at least 1 test gave a positive result, the patient was classified as COVID19 positive, and the date of their earliest positive COVID-19 test result was used as their baseline date. For patients with multiple COVID-19 tests that were all negative, the patient was classified as COVID-19 negative, and the date of their earliest negative COVID-19 test results was used as their baseline date. Patients were tested in the context of seeking care for COVID-19; the tests were not part of general screening and surveillance.

Only patients with at least 180 days of follow-up time after their baseline date were retained in the cohort. Patients with more than 180 days of followup were censored at 180 days. Mortality data were available in the databank, and patients who died within their 180-day window were included in the analysis and censored at the date of their recorded death. Mortality data were obtained from both patient electronic health records and the Social Security Death Index.

COVID-19-positive patients were also categorized as having had either a severe or mild/moderate COVID-19. Patients seen only in an outpatient setting were classified as having mild/moderate COVID-19, while those hospitalized for any reason during their first 30 days of follow-up were classified as severe. The cohort was also censored for 30 days after baseline in the COVID-19 negative patients or until 30 days post-hospital discharge for the severe COVID-19 patients to ensure that health care utilization was post-acute and not part of the initial COVID-19 episode of care (eg, readmission).

\section{Outcome Variables}

Based on previous literature regarding organ systems affected by COVID-19 and likely complications, outcomes were determined as the occurrence of hospital admission for a cardiovascular, respiratory, or clotting disorder-related diagnosis. ${ }^{8-10}$ The targeted hospitalizations investigated as cardiovascular outcomes were myocardial infarction, heart failure, and stroke. The respiratory hospitalizations were pneumonia, hypoxemia, and acute respiratory distress syndrome. The clotting disorder hospitalizations were deep vein thrombosis, venous thromboembolism, and pulmonary embolism.

The International Classification of Diseases, Tenth Version (ICD-10) codes used to define these outcomes are given in Table 1. Outcomes were assessed as hospitalization for any of these causes and by category. In the combined analysis, the earliest occurrence of admission for a cardiovascular, respiratory, or clotting disorder complication was used for patients who experienced multiple hospitalizations during the follow-up time frame.

Because COVID-19 may lead to hospitalizations for other causes than just the ones we have identified that have a plausible mechanism, we also examined hospitalizations for any cause. In the all hospitalizations analysis, a patient could have had any diagnosis associated with their hospitalization, including those not listed in Table 1. 
Table 1. Diagnosis Codes for Analyzed Outcomes and Comorbidities

\begin{tabular}{ll}
\hline Outcome conditions & ICD-10/ICD-9 Codes \\
\hline Cardiovascular Diagnoses & \\
Heart failure & I21 \\
Myocardial infarction & I50, I11.0, I13.0, I13.2 \\
Stroke & G46, I63, I69 \\
Respiratory Diagnoses & J12, J13, J15, J16, J17, J18, \\
Pneumonia & J82, J84 \\
& J80, J96 \\
Acute respiratory distress & R09.02 \\
syndrome & \\
Hypoxemia & Q26, I82 \\
Clotting Diagnoses & \\
Deep vein thrombosis/ & I26, I74, I82, J81.1 \\
Venous thromboembolism & \\
Pulmonary embolism & I10, 401 \\
Comorbidities & E11.0, E11.2, E11.3, E11.5, \\
Hypertension & E11.6, E11.8, E11.9, \\
Diabetes mellitus & E11.40, E11.41, E11.43, E11.49, 250.00, \\
& $250.02,250.2,250.3$, \\
& $250.4,250.5,250.6,250.7$, \\
COPD/Asthma & $250.8,250.9$ \\
\hline & $\mathrm{J} 43, \mathrm{~J} 44, \mathrm{~J} 45,491.2,492,493$ \\
\hline
\end{tabular}

COPD, chronic obstructive pulmonary disease; ICD-10/ICD9, International Classification of Diseases, Tenth Version and Ninth Version.

\section{Comorbidities}

Comorbidities and demographic variables which could potentially confound the associations between mild/moderate COVID-19, severe COVID-19, and no COVID-19 and the risk of hospitalization for post-acute COVID-19 complications were collected at baseline for each member of the cohort. Demographic variables included patient age, race, ethnicity, sex, and insurance status. Comorbidities included hypertension, chronic obstructive pulmonary disease (COPD)/asthma, diabetes mellitus, and overweight/obesity. Hypertension, COPD/asthma, and diabetes mellitus were determined using ICD-9/10 codes (Table 1), and a patient's status as overweight/ obese was determined using weight and height measurements available in the EHR. Body mass index (BMI) in $\mathrm{kg} / \mathrm{m}^{2}$ was recorded at baseline or the closest available measurement in time.

\section{Analysis}

Hazard ratios for the risk of hospitalization for post-acute COVID-19 complications by COVID-
19 status were determined using Cox proportional hazard models. We obtained hazard ratios for hospital admissions based on COVID-19 diagnosis category, using COVID-19 negative status as the reference. These analyses were then modified to control for age, sex, race, insurance status, and comorbidities. The proportional hazards assumption was confirmed by inspecting the Schoenfeld residual plots for each variable included in the models and testing the time-dependent $\beta$ coefficients. Analyses were conducted using the survival package in $\mathrm{R} v 4.0 .3$.

\section{Results}

A total of 10,646 patients were included in the final cohort. The characteristics of the sample split into the 3 groups of (a) mild/moderate COVID-19, (b) severe COVID-19, and (c) negative COVID-19 is shown in Table 2. Figure 1a presents the KaplanMeier curves comparing the risk of hospitalization for all conditions between all patients who were COVID-19 positive and those who were COVID19 negative. Using Cox regression, the hazard ratio for the risk of hospitalization for any condition among those who were COVID-19 positive was not significantly increased in comparison to those who were COVID-19 negative (HR, 1.10; 95\% CI, 0.84-1.45). After adjustment for potential confounding variables, the results still indicated no significantly increased risk in future hospitalization for any condition for patients who were COVID-19 positive versus those who were COVID-19 negative (HR, 1.31; 95\% CI, 0.98-1.74).

Figure 1b shows a Kaplan-Meier curve of the unadjusted survival probabilities for hospitalizations over time between the severe COVID-19 group and the negative COVID-19 group. The risk of hospitalization post-COVID-19 infection is presented in Table 3. In the unadjusted analyses, severe COVID-19 infection has a significantly increased risk compared with those with no COVID-19. Although the unadjusted analyses suggest that mild/ moderate COVID-19 may have a decreased risk for experiencing a post-acute hospitalization for any condition compared with no COVID-19, the effect is no longer significant after adjustment for potential confounding variables. In adjusted analyses, severe COVID-19 has a significantly increased risk of hospitalization compared with the patients who were COVID-19 negative. Further, the severe 
Table 2. Characteristics of the COVID-19-Positive and COVID-19-Negative Patients in the Cohort

\begin{tabular}{|c|c|c|c|c|c|}
\hline & $\begin{array}{c}\text { Total } \\
(\mathrm{N}=10,646) \\
\end{array}$ & $\begin{array}{c}\text { Severe COVID-19 } \\
(\mathrm{N}=114) \\
\end{array}$ & $\begin{array}{l}\text { Mild/Moderate COVID-19 } \\
(\mathrm{N}=211) \\
\end{array}$ & $\begin{array}{l}\text { No COVID-19 } \\
(\mathrm{N}=10,321) \\
\end{array}$ & $P$ value* \\
\hline & n (\%) & n (\%) & n (\%) & $\mathrm{n}(\%)$ & \\
\hline Any hospitalization & $1626(15.3)$ & $34(29.8)$ & $18(8.5)$ & $1574(15.3)$ & $<.001$ \\
\hline $\begin{array}{l}\text { Combined group of } \\
\text { hospitalizations }\end{array}$ & $891(8.4)$ & $27(23.7)$ & $14(6.6)$ & $850(8.2)$ & $<.001$ \\
\hline $\begin{array}{l}\text { Cardiovascular } \\
\text { hospitalizations }\end{array}$ & $637(6.0)$ & $21(18.4)$ & $9(4.3)$ & $607(5.9)$ & $<.001$ \\
\hline $\begin{array}{l}\text { Respiratory } \\
\text { hospitalizations }\end{array}$ & $491(4.6)$ & $17(14.9)$ & $7(3.3)$ & $467(4.5)$ & $<.001$ \\
\hline $\begin{array}{l}\text { Clotting } \\
\text { hospitalizations }\end{array}$ & $195(1.8)$ & $8(7.0)$ & $2(0.9)$ & $185(1.8)$ & $<.001$ \\
\hline Male & $4329(40.7)$ & $47(41.2)$ & $79(37.4)$ & $4203(40.8)$ & .626 \\
\hline Non-Hispanic White & $6713(63.1)$ & $46(40.4)$ & $96(45.5)$ & $6571(63.7)$ & $<.001$ \\
\hline Non-Hispanic Black & $2560(24.0)$ & $55(48.2)$ & $61(28.9)$ & $2444(23.7)$ & $<.001$ \\
\hline Hispanic & $640(6.0)$ & $7(6.1)$ & $28(13.3)$ & $605(5.9)$ & $<.001$ \\
\hline \multicolumn{6}{|l|}{ Age, years } \\
\hline $18-29$ & $1309(12.3)$ & $10(8.8)$ & $50(23.7)$ & $1249(12.1)$ & $<.001$ \\
\hline $30-44$ & $2119(19.9)$ & $13(11.4)$ & $46(21.8)$ & $2060(20.0)$ & .059 \\
\hline $45-64$ & $3847(36.1)$ & $29(25.4)$ & $75(35.5)$ & $3743(36.3)$ & .056 \\
\hline 65 and older & $3371(31.7)$ & $62(54.4)$ & $40(19.0)$ & $3269(31.7)$ & $<.001$ \\
\hline Insurance & $10,312(96.9)$ & $114(100.0)$ & $194(91.9)$ & $10,004(96.9)$ & $<.001$ \\
\hline Diabetes mellitus & $2820(26.5)$ & $64(56.1)$ & $45(21.3)$ & $2711(26.3)$ & $<.001$ \\
\hline Hypertension & $5719(53.7)$ & $89(78.1)$ & $88(41.7)$ & $5542(53.7)$ & $<.001$ \\
\hline COPD & $3174(29.8)$ & $39(34.2)$ & $36(17.1)$ & $3099(30.0)$ & $<.001$ \\
\hline Overweight & $2714(25.5)$ & $30(26.3)$ & $61(28.9)$ & $2623(25.4)$ & .504 \\
\hline Obese & $3813(35.8)$ & $41(36.0)$ & $88(41.7)$ & $3684(35.7)$ & .197 \\
\hline
\end{tabular}

COVID-19, Coronavirus disease 2019.

*Results from $\chi^{2}$ tests.

COVID-19 group had a significantly increased risk of hospitalization compared with the mild/moderate COVID-19 group in adjusted analyses. The mild/ moderate COVID-19 patients were not at increased risk of future hospitalization compared with the COVID-19 negative group.

\section{Discussion}

These results indicate a substantial impact on the likelihood of severe post-acute COVID-19 sequelae depending on the severity of the initial COVID-19 episode. Even though the results do not show that COVID-19 infection increases the likelihood of future hospitalization, the likelihood of hospitalization 6 months following a severe COVID-19 is greatly increased above COVID-19 negative patients and those who had mild/moderate COVID-19. Importantly, mild/moderate COVID-19 patients did not have significantly different risks for a subsequent hospitalization than COVID-19-negative patients.
This study adds to the accumulating literature of post-acute sequelae following a COVID-19 infection. Other studies have focused on important but less severe complications than hospitalization, which was the focus of the present study. ${ }^{4}$ Further, the present study also provides evidence of complications of COVID-19 infection in comparison to patients who were COVID-19 negative, allowing an increased understanding of the impact of COVID-19. According to the Centers for Disease Control and Prevention, between August 1, 2020, and April 5, 2021, there have been nearly 2 million $(>1,978,000)$ COVID-19 confirmed hospitalizations in the United States. ${ }^{14}$ These numbers indicate the large group of patients who are at increased risk for a significant COVID-19 complication post-hospitalization. As we discuss flattening the curve to decrease hospitalizations for COVID19 , this data provides new evidence of the association of COVID-19 with future hospitalizations that would not be diagnosed or counted as COVID-19. 
Figure 1. a. Kaplan-Meier Curve Comparing All Hospitalizations Between COVID-19-Positive and CoVID-19Negative Patients.

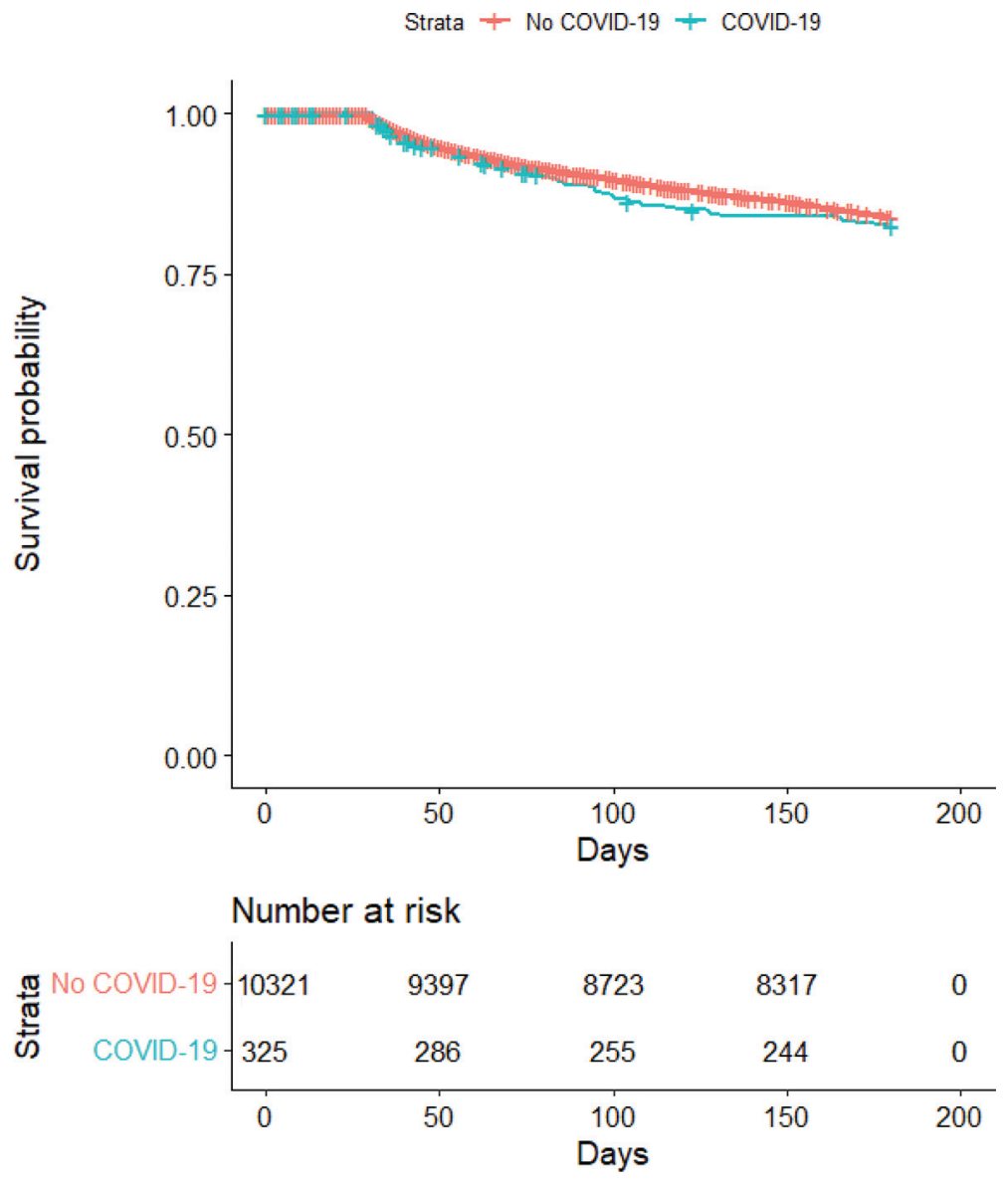

The lack of a significantly increased risk of hospitalization among mild/moderate COVID-19 patients is encouraging. Increased vaccination for COVID-19 and mitigation strategies have played roles in decreasing new cases, and these results elevate the importance of vaccinating patients at risk for severe COVID-19.

There are several limitations to this analysis that need to be considered. First, the analysis was based on patients seen in 1 health system with a regional catchment area. Although more than 10,000 PCRbased COVID-19 diagnoses were included in the analysis, the study cohort may not represent the patient population in other areas of the United States. Second, we focused on hospitalizations as our outcome of interest. We focused on hospitalizations as a priority outcome due to the gap in our knowledge of downstream complications of COVID-19 and the serious impact of hospitalizations on both the patient and the health system, but other outcomes may exhibit different patterns.
Third, we are not able to determine the reasons that patients chose to interact with our health system. It is possible that asymptomatic patients who had not been exposed to the virus sought a COVID-19 test before traveling and were included in the analysis. We were also unable to determine whether patients were residents of a nursing home or long-term care facility, potentially affecting the estimated hazard ratios. Finally, the analyses comparing different severity of COVID-19 may have been affected by limited sample sizes. It should be noted that statistically significant effects were found for several outcomes comparing mild/moderate to severe COVID-19.

In conclusion, COVID-19 has significant postacute sequelae. Patients with a severe episode of COVID-19 have a significantly increased likelihood of hospitalization over the next 6 months for conditions other than COVID-19. These results reinforce the importance of vaccination and mitigation strategies for patients at high risk for a severe COVID-19 episode. 
Figure 1. b. Kaplan Meier Curve Comparing Combined Hospitalizations Between Severe CoVID-19-Positive and COVID-19-Negative Patients.

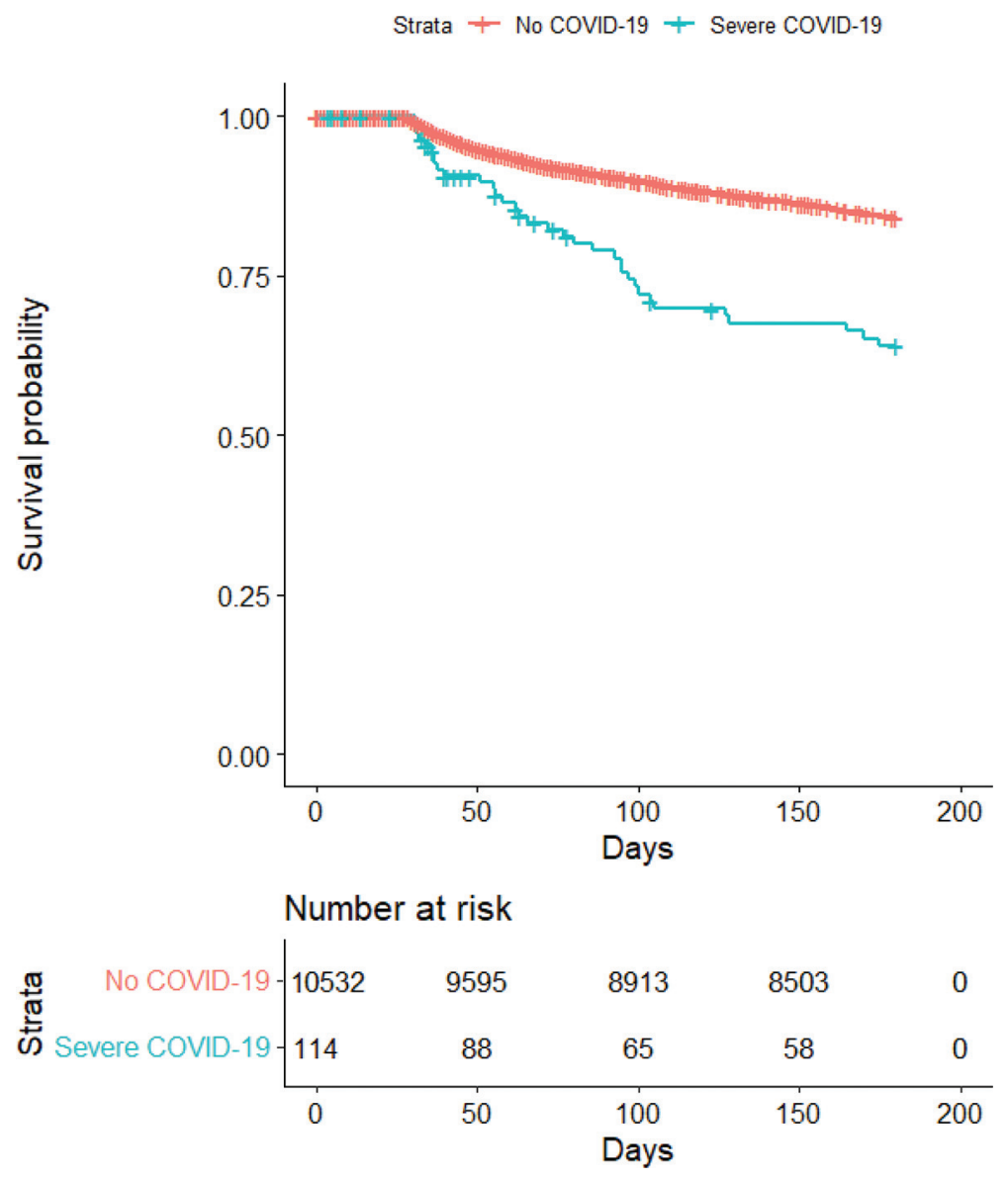

Table 3. Risk of Future Hospitalization by COVID-19 Status for Conditions Other than CoVID-19

\begin{tabular}{|c|c|c|c|c|c|c|}
\hline & \multicolumn{6}{|c|}{ Hazard Ratios (95\% CI) } \\
\hline & \multicolumn{3}{|c|}{ Unadjusted } & \multicolumn{3}{|c|}{ Adjusted $^{*}$} \\
\hline & $\begin{array}{l}\text { Mild/Moderate } \\
\text { versus No } \\
\text { COVID-19 }\end{array}$ & $\begin{array}{c}\text { Severe versus } \\
\text { No COVID-19 }\end{array}$ & $\begin{array}{l}\text { Severe versus } \\
\text { Mild/Moderate } \\
\text { COVID-19 }\end{array}$ & $\begin{array}{l}\text { Mild/Moderate } \\
\text { versus No } \\
\text { COVID-19 }\end{array}$ & $\begin{array}{c}\text { Severe versus } \\
\text { No COVID-19 }\end{array}$ & $\begin{array}{l}\text { Severe versus } \\
\text { Mild/Moderate } \\
\text { COVID-19 }\end{array}$ \\
\hline Any hospitalization & $0.53(0.33-0.85)$ & $2.55(1.82-3.58)$ & $4.80(2.71-8.50)$ & $0.71(0.44-1.17)$ & $2.16(1.53-3.04)$ & $3.01(1.66-5.48)$ \\
\hline $\begin{array}{l}\text { Combined targeted } \\
\text { hospitalizations }^{\dagger}\end{array}$ & $0.78(0.46-1.33)$ & $3.73(2.54-5.48)$ & $4.76(2.50-9.08)$ & $1.02(0.59-1.76)$ & $2.24(1.52-3.30)$ & $2.20(1.13-4.28)$ \\
\hline $\begin{array}{l}\text { Cardiovascular } \\
\text { hospitalization }\end{array}$ & $0.71(0.37-1.37)$ & $4.05(2.62-6.25)$ & $1.41(0.73-2.72)$ & $0.92(0.46-1.86)$ & $2.30(1.48-3.57)$ & $2.50(1.10-5.66)$ \\
\hline $\begin{array}{l}\text { Respiratory } \\
\text { hospitalization }\end{array}$ & $0.72(0.34-1.51)$ & $4.09(2.52-6.64)$ & $5.72(2.37-13.78)$ & $1.05(0.50-2.23)$ & $2.64(1.62-, 4.31)$ & $2.51(1.03-6.07)$ \\
\hline $\begin{array}{l}\text { Clotting } \\
\text { hospitalization }\end{array}$ & $0.52(0.13-2.09)$ & $4.86(2.39-9.86)$ & $9.36(1.99-44.08)$ & $0.64(0.16-2.60)$ & $3.32(1.62-6.80)$ & $5.17(1.09-24.50)$ \\
\hline
\end{tabular}

COPD, chronic obstructive pulmonary disease; COVID-19, Coronavirus disease 2019; CI, confidence interval.

*Models were adjusted for age, race/ethnicity, diabetes mellitus, hypertension, COPD, overweight/obesity, sex, and insurance status.

${ }^{\dagger}$ Combined cardiovascular, respiratory, and clotting hospitalizations. 
To see this article online, please go to: bttp://jabfm.org/content/ 34/5/907.full.

\section{References}

1. Dong E, Du H, Gardner L. An interactive webbased dashboard to track COVID-19 in real time. Lancet Infect Dis 2020;20:533-4.

2. Garg S, Kim L, Whitaker M, et al. Hospitalization rates and characteristics of patients hospitalized with laboratory-confirmed Coronavirus disease 2019 - COVID-NET, 14 states, March 1-30, 2020. MMWR Morb Mortal Wkly Rep 2020;69: 458-64.

3. Del Rio C, Collins LF, Malani P. Long-term health consequences of COVID-19. JAMA 2020;324: $1723-4$.

4. Logue JK, Franko NM, McCulloch DJ, et al. Sequelae in adults at 6 months after COVID-19 infection. JAMA Netw Open 2021;4:e210830.

5. Huang C, Huang L, Wang Y, et al. 6-month consequences of COVID-19 in patients discharged from hospital: a cohort study. Lancet 2021;397:220-32.

6. Cortinovis M, Perico N, Remuzzi G. Long-term follow-up of recovered patients with COVID-19. Lancet 2021;397:173-5.

7. Janiri D, Carfi A, Kotzalidis GD, et al. Posttraumatic stress disorder in patients after severe COVID-19 infection. JAMA Psychiatry 2021;78:567-9.
8. Zuin M, Rigatelli G, Zuliani G, Roncon L. The risk of thrombosis after acute-COVID-19 infection. QJM 2021;hcab054.

9. Umbrajkar S, Stankowski RV, Rezkalla S, Kloner RA. Cardiovascular health and disease in the context of COVID-19. Cardiol Res 2021;12:67-79.

10. Brosnahan SB, Jonkman AH, Kugler MC, Munger JS, Kaufman DA. COVID-19 and respiratory system disorders: current knowledge, future clinical and translational research questions. Arterioscler Thromb Vasc Biol 2020;40:2586-97.

11. Lavery AM, Preston LE, Ko JY, et al. Characteristics of hospitalized COVID-19 patients discharged and experiencing same-hospital readmission - United States, March-August 2020. MMWR Morb Mortal Wkly Rep 2020;69:1695-9.

12. Parra LM, Cantero M, Morrás I, Puerta de Hierro Hospital Admission Study Group, et al. Hospital readmissions of discharged patients with COVID19. Int J Gen Med 2020;13:1359-66.

13. Rokadiya S, Gil E, Stubbs C, Bell D, Herbert R. COVID-19: Outcomes of patients with confirmed COVID-19 re-admitted to hospital. J Infect 2020;81:e18-e19.

14. Centers for Disease Control and Prevention. COVID-NET. Laboratory-Confirmed COVID19-Associated Hospitalizations. Available at: https:// covid.cdc.gov/covid-data-tracker/\#new-hospitaladmissions. Accessed April 8, 2021. 